teeth in the young and the old membrane. Like all cellular elements of the membrane, they grow less numerous with age, but they have been seen in the membrane from a man 70 years old.

The size, number, persistence, and conspicuousness of

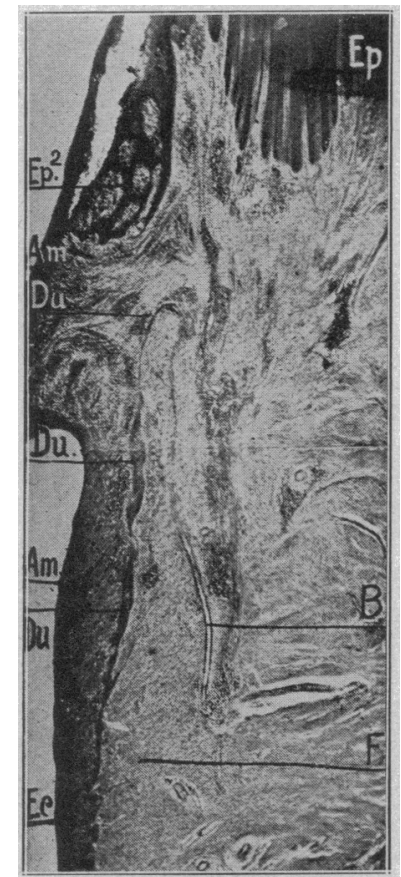

Longitudinal section of duct-like structure. Rephotographed from two photomicrographs with an A. A. Zeiss obj. Ep, epithelium covering the gum. Ep 2, epithelium lining the gingivus in the region of the gingival gland. Cm, cementum which has separated from the dentine in fibrous tissue of the membrane.

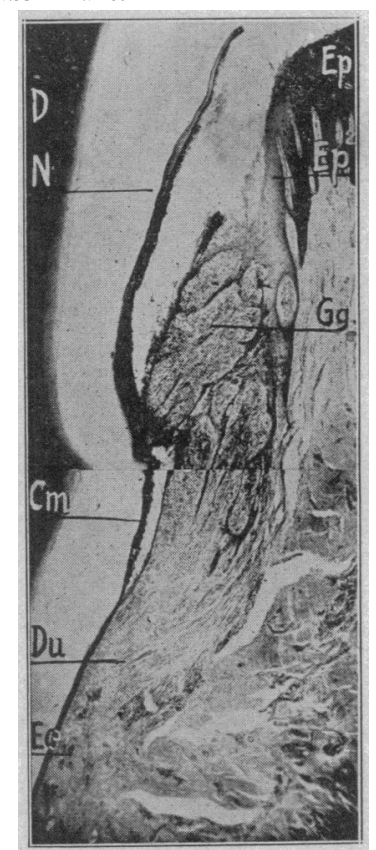

Longitudinal section showing the gingival gland. Rephotographed from two photomicrographs made with an A. A. Zeiss obj. Ep, epithelium covering the gum. Ep 2, epithelium lining the gingivus. Gg, gingival gland showing the round cells between the epithelial legs. N, Nasmyths' membrane. Cm, cementum separated from the dentine by decalcification. Du, bit of a duct. Ec, epithelial cords.

these structures make it seem extremely improbable that they are simply embryonal remains from the epithelial cord, or external or internal tunics of the enamel organ, as suggested by the work of Von Brom, quoted by Charles Thomas ${ }^{2}$, and as was suggested to me by Dr.

\footnotetext{
2 Dental Anatomy. 107.
}

Huber of Ann Arbor. I have not attempted to trace the origin of them, but in the last few weeks, by the kindness: of Dr. John Palmer of Chicago, I obtained some rabbits' jaws, soon after birth. I have studied them over, and have been unable to find any trace of such structure in membranes of unerupted teeth. It seems to me that these structures must be present for a purpose. What that may be I cannot suggest, and I know nothing that. throws any light on the question.

In order to work out the problem to meet the requirements of the histologist, four things must be done.

1. The origin of the structures must be traced, so as to determine from what tissue they are derived.

2. 'Their relation to the blood-supply must be determined.

3. Their morphology must be determined, by making a complete series of sections, both longitudinal and transverse, to determine whether they have ducts or not, or other connection with the gingival epithelium; and the complete reconstruction of them from serial section. The last task is perhaps impossible, but it could be done for small areas so as to satisfy the demand.

4. Their condition in diseased conditions of the membrane must be carefully studied.

Until such a program is followed out we can but speculate as to what the origin and function may be, and speculation of this kind does not often aid in the advancement of scientific knowledge.

\section{SYPHILIS IN SURGICAL PRACTICE.}

BY ARTHUR DFAN BEVAN, M.D.

Surgeon to Presbyterian Hospital; Associate Professor of Surgery, Rush Medical College. CHICAgo.

My father was a physician. One day, when a boy of 16 , I was in my father's office when a tall, fine-looking man of 35 or 40 came in to consult. him. This man was a German-American of considerable prominence. I had often met him before, and had always admired him. He was a great thick fellow, more than six feet tall, with large brown shaggy head and beard, deep-voiced, bright-eyed, the picture of physical and mental health; a fine type of the Teutonic massiveness and vitality that has relegated the Latin races to the dying nations of the world. I remember that when I read "Cæsar's Commentaries," with the description of the giant Germans, who disputed Cæsar's advance across the Rhine, and who died so bravely, crushed by the better arms and better discipline of the Roman legions, I thought of this man; they were his ancestors. He was a picture of what they had been. After he had left the office I drove with my father on his afternoon round of visits. We had been in the buggy, driving possibly ten minutes, when my father suddenly turned around to me and, for the first time in my life, gave me some advice on the subject of sexual intercourse. He told me of the penalties of promiscuous intercourse, the virulence of gonorrhea, the ravages of syphilis. He drew a picture of an invalid wife, a blind child, the man with the blurred brain and the incoherent speech of syphilitic aphasia, and the staggering gait of ataxia. He was a graphic word-painter, and I remember as a boy it made a profound impression on me. And in some way I always associated this, my first knowledge of venereal disease, with the German who had left the office but a short time before. That was twenty-two years ago. Two weeks ago an old man, of great massive frame, dragging his *Read before the Alumni Association of Rush Medical College, May 24, 1899. 
right leg, shuffled unsteadily into my office; his pocket handkerchief and gloves were almost falling out of his right overcoat pocket; he could not articulate above a whisper, and even then could command but a few words. The left eye was dulled with an old iritis; the right eye was reddened by an acute attack; the pupil dilated unevenly with atropin; the hands presented syphilitic skin and nail lesions; and this wreck was my German, the German whom I had idealized; plus syphilis. With some difficulty I obtained his history, and, most interesting of all, the fact that he had always denied to the many physicians who had treated him for twenty years for various lesions, principally if not entirely syphilitic, syphilis. My father and myself, he said, were the only ones who knew that he had syphilis, because, as he stated, my father had told him at the end of two years' treatment that he regarded him as cured. My father died about that time, and he said no one then living knew that he had had syphilis, and he had a family and he could not have anyone know, and would I swear to him that I would, not tell any one? You are all familiar with the picture I have so crudely drawn, the common picture of syphilis and its concealment, interesting only because of the frame of circumstances which surrounds it.

When I received my degree in medicine $I$ accepted a contract position as acting assistant-surgeon in the U. S. Marine-Hospital service. I was stationed at the marine hospital in Chicago. My duties were to look after the out-patient department of the work. In this department we treated nearly 3000 patients annually, about one-third of these being venereal diseases, and of these 1000 venereal cases, many were cases of syphilis, representing all stages of the disease. Syphilis became the first great problem in disease with which I wrestled, and the diagnosis, the vagaries, the treatment, and the tenacity of the lesion became an interesting study, for which later five years of regular service in marine hospitals afforded an abundance of material. The sailor man is as a class easily the point of least resistance in the community to syphilis. His long voyages and enforced continence, and his drunken embrace of blear-eyed Venus during his short spree in port, make him an almost certain victim of infection, and the fact that he seldom early receives the benefit of proper and continued medication enables the marine surgeon, into whose hands the derelict finally drifts, to study often the natural history of syphilis unmodified by treatment. Many of our cases are honestly ignorant of having syphilis; some would deny and later confess to a syphilitic history, when confronted with positive evidence. I early learned to place little or no reliance on the previous history in making a diagnosis.

Since leaving the marine-hospital service, ten years ago, I have devoted my time exclusively to general surgery, and in this work I have found that my early syphilitic training has been of great service. I do not mean to pose as a syphilographer, but I am sure that I have been saved many mistakes by this training. I am sure that $I$ have seen many cases which this training clearly branded syphilis to me, overlooked by the specialist, the surgeon and the general practitioner. On the other hand, I must confess that in a number of cases I have believed lesions syphilitic which were later proved to be of other origin. I have learned to know that syphilis is not a respecter of class or person-rich and poor, youth and age, priest and libertine, prostitute and virgin, guilty and innocent, all can and do acquire this disease. We must not be astonished in finding it anywhere. We must never accept a negative history as definite evidence of its absence. Syphilis is more widespread than is generally supposed. It causes many an illness when it is not recognized or even suspected. Without carrying our suspicion to extreme, we should constantly be on the watch for syphilis in our everyday work.

It is not my intention to present a discussion of typical syphilis, as is done by the syphilographers, in textbooks, but believing that a broad discussion of the unusual and often unrecognized lesions of syphilis as they present themselves to the general practitioner and specialist might interest all, I will open the discussion by reviewing some of my experiences with syphilis in surgical work. I might entitle this brief paper, then, "Unusual Syphilis in Surgical Practice."

The subject of extragenital chancre will furnish the unusual form in the primary stage. I have handled about fifteen of these lesons which I can now call to mind; most interesting to us possibly are the extragenital chancres which physicians acquire in their professional work. I have seen a number of these cases; the majority of them presented none of the characteristics of chancre and were mistaken for pus infections, or tuberculous infections, and later, when the axillary lymphatics were involved and a low form of syphilitic fever was present, the axillary lymphatics were removed; the subsequent development of the secondary symptoms cleared up the diagnosis. Several of my cases have been seen by expert syphilographers, and the primary lesion positively pronounced not the initial lesion of syphilis. In one case an expert said that if it was a chancre it certainly had none of the characteristic signs, and that he had never seen one like it. In another case, seen by me with a colleague, the syphilographer staked his professional reputation that the lesion was not specific, and yet in both, the later secondary symptoms disproved their statements. In both these cases the axillary lymphatics were removed with the idea that we had a low form of pus infection or mixed infection to deal with. The lesson to be learned from such cases is that extragenital chancres of the fingers are seldom typical. An infection on a physician's hand, which is not acute, which produces enlargement of the axillary lymphatics, which persists for more than ten days, which is locally limited, which is accompanied by a slight continued elevation of temperature, is suspicious of syphilis, no matter what the size, shape, or consistency of the sore may be.

In looking into this question I find that trained nurses very rarely acquire syphilis of the fingers. Among 500 nurses observed from 1883-1898, no cases; among physicians it is much more common than is generally believed. Among the several hundred physicians, certainly less than five hundred of my acquaintance, I know of six cases of extragenital chancre. This is almost entirely preventable and shows the necessity of the observance of the simple prophylactic rules required, and evidently followed out more carefully by nurses than physicians. The primary sore of the finger may be so small and so transient that it is not recognized; the secondarv symptoms following may be slight and not interpreted and the case goes on to tertiary syphilis untreated. I have seen this in the case of a physician of unusual intelligence, who, in the light of tertiary symptoms, was able to review in this new light his previous four years and could plainly recall the primary sore and the mild secondary symptoms. In the light of this case, I have no doubt that many cases of syphilis exist in the com- 
munity umrecognized by the physician and unknown to the patient. Extragenital chancres of the lips are fairly common; usually the infection is carried from a mucous patch on the lip of the infector; sometimes, of course, due to sexual perversion, and sometimes due to an indirect infection from instruments in the hands of dentists or physicians.

I have had one chancre of the tonsil occurring in the case of a physician who had his throat treated by a throat specialist, the infection being carried by the instruments used. A great mass of glands on the side of the neck followed the small unrecognized chancre. Two prominent surgeons diagnosed tuberculosis of the cervical glands and advised removal. Prof. James Nevins Hyde and myself suggested the possibility of syphilis. The doctor patient ridiculed the idea, but decided to await developments. Within a few weeks secondary symptoms occurred; treatment, rapid disappearance of glands, but later, unfortunately, brain syphilis.

An unusual type of extragenital syphilis occurred in a doctor who presented himself with the history: He tripped and fell on an iron rail, cut the skin over the patella; wound slow in healing; examined and probed by a number of doctors; wound almost healed, and then atter twenty days a circular hard ulcer developed at the site of traumatism; hard chancre from infected instruments or fingers.

A physician brought me to see his son-in-law, his grandchild and his wife, who he said had been poisoned by poison ivy. The man had a pustular syphilide, the child inherited syphilis of the skin and skeleton, the grandmother a history of having had a sore on the arm on which she usually carried the child. This healed slowly, was accompanied by weakness and deterioration of general health, and she was, when I saw her, suffering from a macular syphilide. The mother of the child had no symptoms and my doctor friend, the grandfather, has escaped.

When stationed in Portland, Ore., my position as marine surgeon brought me many patients from the salmon fishermen; some seven thousand were at the mouth of the Columbia River. These men, like sailors, frequently acquire syphilis. Two of them go in a boat to tend their nets and live together for many weeks and months. Sodomy is common, and as a result chancre, chancroid and gonorrhea of the rectum occur. I have seen several cases of chancre of the rectum among these men, but have never been able to follow out the later history of these cases to determine whether the lesion was the initial lesion of syphilis or chancroid.

The facts to be kept in mind in extragenital chancre are: 1 , its atypical character; 2 , that it is common, at least that it is not a rare lesion; 3 , that it is possible in any individual; 4 , that we should wait for secondary symptoms before placing the patient on constitutional treatment.

The second unusual form of syphilis I desire to mention is the unusually severe constitutional symptoms called syphilitic fever. I have seen one case in which $I$ believe that it is possible that death resulted from the profound primary syphilitic intoxication before secondary symptoms developed; history of healing, hard chancre, bubo, severe pains in limbs, high continued fever, and death at the end of ten days; no post-mortem made. I have diagnosed typhoid several times and later found that I had syphilitic fever to deal with.

I shall pass over the unusual skin lesions of secondary syphilis because I am not qualified to discuss them. I shall simply remark, in passing, that I am impressed with the fact that they may simulate almost any skin lesion, and that I lose confidence in the dermatologist who with great emphasis affirms positively that a certain lesion cannot be syphilis because it lacks this or that characteristic of form, color, consistence, symmetry, ete., because $I$ have seen a number of cases in which these positive statements were made turn out to be syphilis after all.

Syphilitic ulcerations of the skin and mucous membrane are common. As a rule, in the light of the previous history they are readily recognized. There is a large number of cases, however, in which the patient conceals the history, or is ignorant of the history, and in which there is no other sign to guide the physician. These cases form a large group, and they are frequently mistaken for other lesions. As types, I will cite the following cases :

Mrs. P. was sent to me by a dermatologist, to remove a small epithelioma from the nose. I found a small ulcer with hard base, about the size of a bean, which had existed for several years; it would sometimes scab over, but never heal. She was a woman of refinement, fine social position, and with no specific history. I put her on iodid in large doses, and in two weeks the ulcer healed. I have had two other cases on the nose with the same history and result.

Mrs. R., was sent with diagnosis of epithelioma of scalp or possibly lupus ; mother of eight children, youngest 12 years; good social position; no specific history. The lesion was of that character in which it was impossible to state the pathology positively. Under iodid of potash the lesion healed in three weeks.

I have seen several cases diagnosed as carcinoma of the tongue heal under iodid. In fact, I believe we should make it a rule, before operating on these small ulcerations of skin and mucous membrane, to give the patient the benefit of the doubt and put him or her on a thorough course of iodids.

We will sometimes make the mistake of confounding some other lesion with syphilis. As an example, I recall a case of a man of 50 , with palmar syphilide, history of syphilis, and an ulceration of the finger, the size of a copper cent, of one year's standing. The diagnosis was positively syphilis. A long course of iodids gave no benefit. I removed a section and Prof. L. Hektoen found carcinoma, which we believed began on a syphilitic base.

Syphilitic lesions of the nose and palate are common. I refer to them here because I have met with several unusual cases. One was sent by a rhinologist, with destruction of cartilage and bone; no syphilitic history, course of iodids 8-grain doses producing no benefit. On increasing to 70 -grain doses, the lesion healed. A second case, positively pronounced by rhinologists as non-specific; no specific history; mercury produced no effect; lesion healed under 300 grains a day. A third case: I removed the greater part of the superior maxilla for a dentist; no specific history; wound became indurated and disease began to invade other jaw. Iodid in 40-grain doses produced a cure.

Cases of suppuration of lymphatic glands, where a specific history is denied, are often due to syphilis. As an example, a business man of 40 , with bubo in groin, positively denied venereal disease. The pus was evacuated; sterile; wound healed slowly. I discovered a small ulceration on the nasal septum; put him on iodid; bubo and ulceration of nose healed rapidly. He then confessed to having had syphilis twelve years before.

The subject of large gummata which are mistaken 
for malignant tumors forms a most interesting chapter of unusual syphilis. Esmarch has written extensively on this point, but the value of his work is not widely known. As types:

CASE 1.-A tumor of the breast was diagnosed carcinoma. Amputation of breast was done. A years later a similar tumor appeared on opposite breast; glands in the axilla enlarged; noticed, by accident, a palmar syphilide. Under large doses of iodid the tumor in the breast disappeared. Fividently the first tumor was also specific.

CASE 2.-A man of 40 was sent by Dr. Frankenthal with a tumor in the thigh the size of a fist; malignant in appearance; diagnosis probable sarcoma; no specific history; removed section and found granuloma. Under iodid the tumor disappeared. The patient later acknowledged a specific history.

CASE 3.-A man of 43 presented a tumor in the breast; no specific history; diagnosed sarcoma, which was removed; wound healed, but became indurated. Iodid in large doses; induration melted away, and the patient then acknowledged a specific history.

Gummata mistaken for a variety of lesions form a mixed class. As examples:

CAsE 1.-A patient of 30 , male, married three years, and two healthy children; no specific history; enlarged testicle; suppuration; ulceration of scrotum; removal of the testicle and infected skin; diagnosis, tuberculosis. 'The wound healed. Later there were two hard masses in the penis the size of walnuts; surgeon desired to amputate penis. I removed a section of granulation tissue, put the patient on iodid and the lesion melted away; patient cured, but still denies syphilis.

CASE 2.-Medicolegal case. A brakeman, in a railroad accident, received an injury to the shoulder; paralysis of sternocleidomastoid and trapezius; threatens to sue company; sent to me for examination and report. I found a gumma in the sternocleidomastoid along the course of the spinal accessory nerve. The patient at first denied syphilis. Ender iodid the gumma melted away, and with it the lawsuit and paralysis disappeared.

CAse 3.-A man of 50 ; pain cver gall-bladder; small mass palpable; diagnosis, gall-bladder lesion or carcinoma of liver. On making an exploratory laparotomy I found what I believed to be a gumma of the liver. The patient is still under treatment.

CASE 4.-A patient under mercurial treatment for syphilis developed pain over liver; jaundice. The jaundice disappeared under large doses of iodid; probably obstructive jaundice due to gumma.

I will pass over unusual forms of syphilis of the stomach, small intestines, and colon, of which I believe I have seen examples, because of lack of positive evidence.

The so-called syphilitic stricture of the rectum is unusual only to the men who are not familiar with its frequency. Although these so-called syphilitic strictures are usually due to gonorrhea, true syphilitic strictures occur; I have one case of syphilis of the kidney to report:

A man at St. Elizabeth's Hospital, seen for Dr. Futterer, presented a large kidney mass; explored; greatly thickened edematous capsule, both fibrous and fatty; nothing in the pelvis; two masses in the capsule which Dr. Futterer, from gross appearance, regarded as tubercular. The patient recovered from operation shortly after ; admitted syphilitic history. He was put on iodid. and the kidney mass melted away; patient now well. I believe it was a syphilitic lesion of the kidney.

In order to be brief, I shall omit in my discussion any cases of unusual syphilis of the heart and blood-vessels, but shall hope that cases of this class will be presented by our internists.

The last class of cases which I desire to present comprises cases of unusual syphilis of bone and joints. The relation of syphilis to fractures can be well shown by three cases.

CASE 1.-A man of 24, under treatment for syphilis, has a gumma on the right humerus. On boarding a street-car he feels something give way in the arm and the arm falls helpless to his side. Examination reveals no new point of motion; some swelling; no deformity; no crepitus. The X-ray shows complete fracture of the humerus without displacement. Spontaneous fracture or fracture from muscular contraction is one of the results of syphilis.

CASE 2.-A man of 25 had fracture of both bones of the leg; delayed union; at the end of three months he confided to his surgeon that shortly before being injured he had secondary syphilis. At once placed on constitutional mixed treatment, union rapidly followed.

CAsE 3.--Inherited syphilis of both bones of leg; bent at right angle. I believe the case to be one of late rickets; operated; straightened limb; no union. The patient returned after several months, I operated again, and placed him on iodid and mercury. Rapid bony union followed.

CAsE 4.-An unusual case of double mastoid disease, operated on at interval of six weeks between; was slow in healing; patient admitted syphilis; iodids given; rapid recovery.

I have seen cases of joint syphilis mistaken for tuberculosis and gonorrheal rheumatism. I shall cite but one instructive case.

A man of 23, a large, strong, robust fellow, with carious disease of the left wrist-joint was treated by iodoform injections and resection; not cured. I believed the case to be tubercular and amputated. Later a large gumma of the arm on same side, and a deep ulceration on the skin of the face appeared. He previously denied syphilis, but now admitted it. Under iodid treatment the lesions disappeared. I have no doubt but that the wrist lesion was syphilitic.

Before closing this rapid and incomplete sketch, which is meant simply as an introduction to a general discussion by the members of the Association, let me call the attention of the Association to the fact which has impressed me more and more as I accumulate experience in the use of iodid of potash in syphilis, and it is this: The fact that a granulation melts away under iodid does not prove absolutely that it is syphilitic. Why? Because we now know that at least one other form of granulation tissue is often similarly absorbed-i. e., actinomycosis, and I had a few months ago under observation a case of blastomycetic dermatitis which undoubtedly disappeared in part under iodid.

ReCentur the Duke of Westminster's horse, Flying Fox, won the Eiclipse stakes at Sandown Park, England, the purse being $£ 10,000-\$ 50,000$. Thus far this item is not in the least medical or of interest to our readers, but when we state that the Duke turned over the whole amount to the Royal Alexandra Hospital, Rhyl, Wales, it becomes most interesting from a medical standpoint. The Lancet, from which we get our information, commends this excellent example to other owners of fast horses who race for love of the sport and not for "filthy lucre." 